\title{
Advances in Muscle Physiology and Pathophysiology
}

\author{
Aikaterini Kontrogianni-Konstantopoulos, ${ }^{1}$ Guy Benian, ${ }^{2}$ and Henk Granzier ${ }^{3}$ \\ ${ }^{1}$ Department of Biochemistry and Molecular Biology, Baltimore School of Medicine, University of Maryland, Baltimore, \\ MD 20742, USA \\ ${ }^{2}$ Departments of Pathology and Cell Biology, Emory University, Atlanta, GA 30322, USA \\ ${ }^{3}$ Department of Physiology and Molecular and Cellular Biology, University of Arizona, Tucson 85721, AZ, USA
}

Correspondence should be addressed to Aikaterini Kontrogianni-Konstantopoulos, akons001@umaryland.edu

Received 6 June 2010; Accepted 6 June 2010

Copyright () 2010 Aikaterini Kontrogianni-Konstantopoulos et al. This is an open access article distributed under the Creative Commons Attribution License, which permits unrestricted use, distribution, and reproduction in any medium, provided the original work is properly cited.

During the recent years, unprecedented amounts of new knowledge have seen the light, revealing key factors that modulate the essential processes of the structure and function of muscle. The assembly of the sarcomeric and extrasarcomeric cytoskeleton, the formation of internal membrane systems and their contribution to the regulation of $\mathrm{Ca}^{2+}$ homeostasis, the molecular dissection of specialized sarcolemmal microdomains, and the anchoring of the intracellular cytoskeletal network with the extracellular matrix are only some of the themes where rapid progress has been made. Advanced genetic, molecular, cellular, biochemical, genomic, and proteomic approaches, using diverse animal models, have unraveled the intricate details of these complex pathways both during health and disease and highlighted new questions and challenges. The breadth of new information has been overwhelming yet exciting, emphasizing once again the absolute need for development of sophisticated, multidisciplinary, and integrated approaches to study the roles of subproteomes rather than individual proteins during development and in adulthood, under normal and stress conditions.

In this volume, we invited authors who study muscle structure and function from different perspectives and angles to contribute original and review articles. A wide array of topics is discussed in this special issue, including the regulated assembly of myofibrils, the biomechanical and biophysical properties of sarcomeric contraction, the dynamic tethering of thin and thick filaments and the unique, compensatory, or even redundant roles of their accessory proteins, the giant sarcomeric proteins, and their roles as molecular blueprints and sensors, the sarcolemma and its role in the mechanical and electrochemical coupling of neighboring cells, the tripartite regulation of $\mathrm{Ca}^{2+}$ cycling orchestrated by the activities of the t-tubules, sarcoplasmic reticulum, and mitochondria, the roles of exercise, fatigue and injury in muscle contraction, and the isolation and use of muscle stem cells. Importantly, many of these processes are altered, compromised, or dysregulated in different forms of skeletal and cardiac myopathies and dystrophies. Some of these are discussed as well.

The editors of this special issue are indebted to all the authors who provided either original data or a comprehensive review of the previous and recent literature, making this special issue appealing to a diverse audience of muscle researchers. Our goal was to touch on different aspects of muscle physiology and pathophysiology at the molecular, cellular, organ, and organismal level. We are delighted to see the outcome of our call in the form of this special volume, and we hope that it will provide a wealthy source of knowledge and information and further stimulate muscle research at all different fronts.

\section{Aikaterini Kontrogianni-Konstantopoulos Guy Benian Henk Granzier}



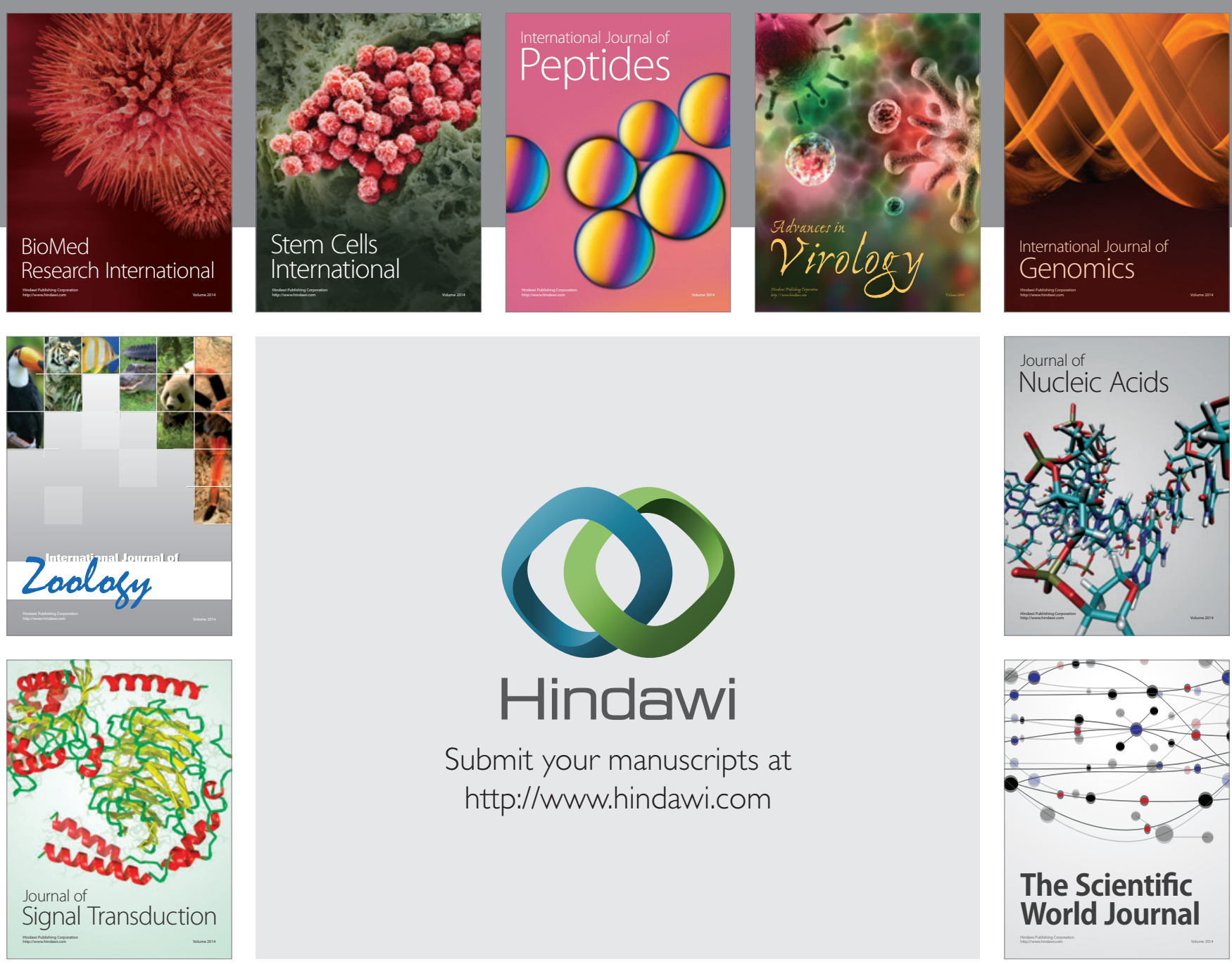

Submit your manuscripts at

http://www.hindawi.com
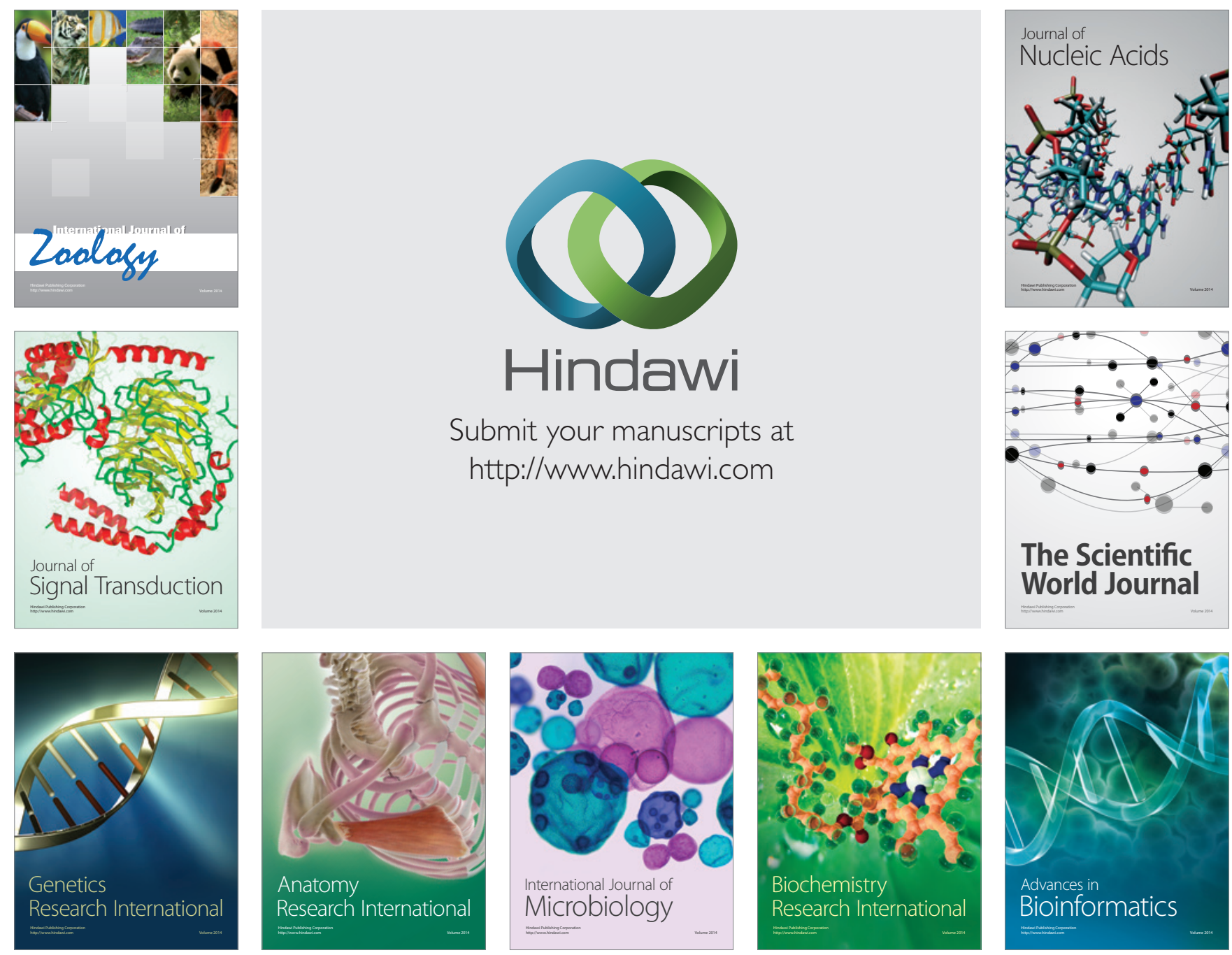

The Scientific World Journal
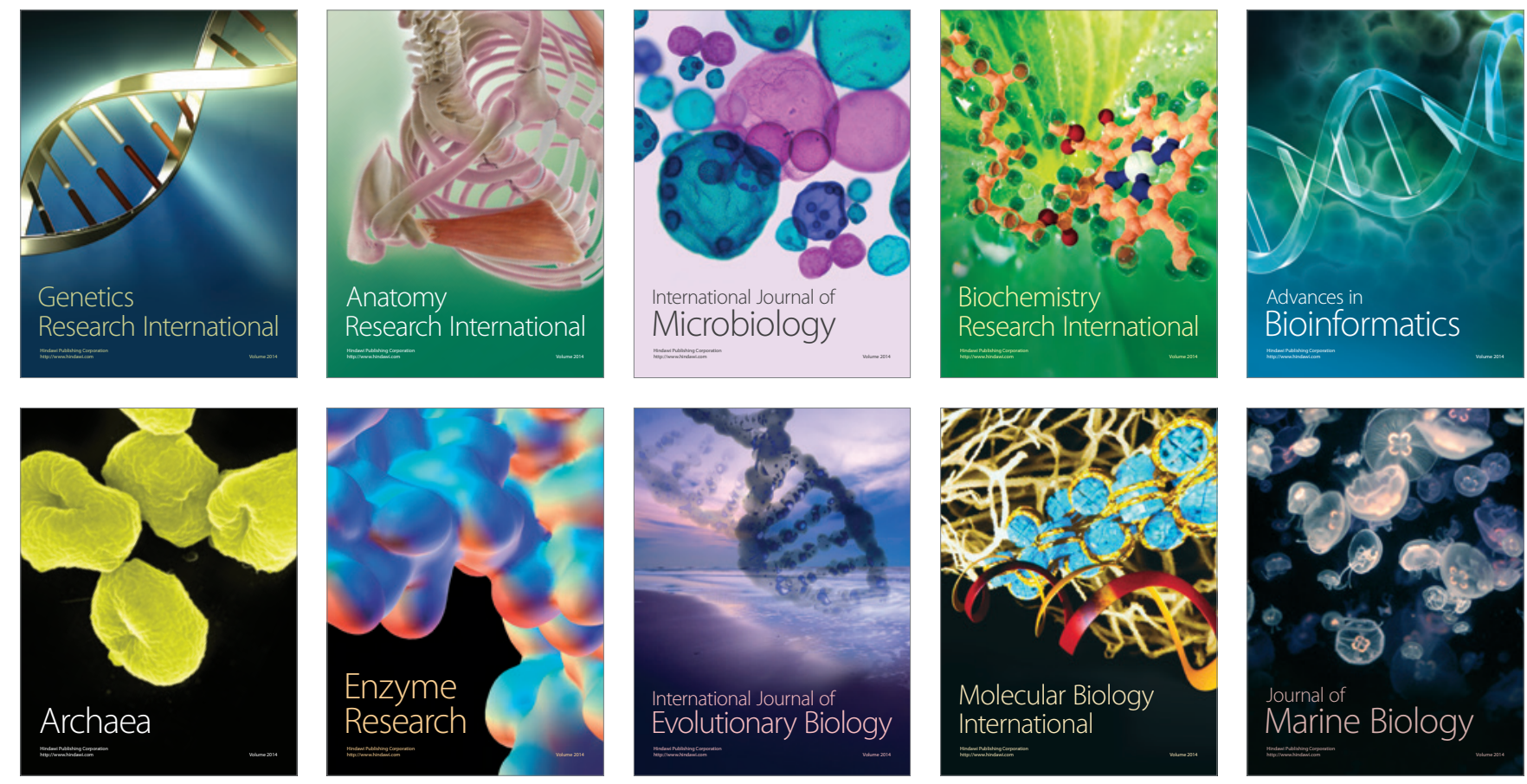